\title{
A parallel algorithm of non-linear high-order dissipative system
}

\author{
Chen Zhao, Zhang Shesheng, Zhao Hualing* ${ }^{*}$ \\ Association of Mathematical Modeling, Wuhan University of Technology, Wuhan, 430070,China \\ ${ }^{a}$ Corresponding author: Zhao Hualing, Zhaohualing@qq.com
}

Keywords: Mathematical model; quantum dynamics, parallel algorithm, Schordinger equation

\begin{abstract}
A parallel computation model is provided of dynamics and dissipative tunneling in a symmetric quartic double well potential. The numerical parallel solution for the position autocorrelation function obtained through the Wigner - Fokker - Planck equation is compared well with numerically exact results.
\end{abstract}

\section{Introduction}

In the field of quantum dynamics, the dissipative systems are of interest for a large variety of problems in physics, chemistry, and biology. The numerically exact solution of the quantum dynamics of dissipative systems remains a challenging problem. Substantial progress has been made during the past decade, and especially noteworthy are the seminal works [1,2]. These include the use of the quantum Wigner - Fokker - Planck equation[3]. This methodology has been developed extensively by Kosloff, Tannor, and co-workers[4].

The main purpose of the present paper is to study numerically what we will term the Wigner Fokker - Planck(WFP) equation.Systematic corrections for this equation have been derived by Tanimura and co-workers[5]. The analytic properties of this equation have been studied, especially by Pechukas and co-workers[6]. We will consider parallel method[7] to solve WFP equation.. .

\section{Basic equation}

.Making $\psi$ is wave function in quantum dynamics, the Schordinger equation can be written as

$$
i \frac{\partial \psi}{\partial t}=\hat{H} \psi
$$

Here the Hamiltonian of the system is described by the (mass weighted) coordinate $\mathrm{x}$ and conjugate momentum p as:

$$
\hat{H}=\hat{H}_{s}+\hat{H}_{b} \quad \hat{H}_{s}=\frac{1}{2} \hat{p}_{0}^{2}+v\left(\hat{x}_{0}\right) \quad \hat{H}_{b}=\frac{1}{2} \sum_{j=1}^{N}\left[\hat{p}_{j}^{2}+\left(\omega_{j} \hat{x}_{j}-\frac{c_{j}}{\omega_{j}} \hat{x}_{0}\right)^{2}\right.
$$

Where $\mathrm{v}$ is potential, the caret serves as a notation for operators, $\hat{H}_{s}$ is system Hamiltonian, $\hat{H}_{b}$ is bath Hamiltonian, cj are coefficient and $\omega \mathrm{j}$ are frequent, subscribe $\mathrm{j}=1, \ldots, \mathrm{N}$. We are interested in the time evolution of an operator at time $t$, defined as:

$$
\hat{O}=\exp (i H t / \hbar) \hat{O} \exp (-i H t / \hbar)
$$

The Wigner representation in the one-dimensional phase space of any operator is

$$
O(p, x, t)=\frac{1}{2 \pi \hbar} \int_{-\infty}^{\infty} e^{i p \varsigma / \hbar}<x-\varsigma / 2|\hat{O}(t)| x+\varsigma / 2>d \varsigma
$$

\footnotetext{
${ }^{1}$ Corresponding author: Zhao Hualing, Zhaohualing@qq.com.
} 
One can then show that the time dependence of the operator in phase space is governed by the Wigner-Liouville equation

$$
\frac{\partial O}{\partial t}=\frac{\partial H}{\partial p} \frac{\partial O}{\partial x}-\frac{\partial H}{\partial x} \frac{\partial O}{\partial p}+W(p, x, t)
$$

The initial condition and boundary condition of above equation are

$$
O(p, x, t=0)=O_{0}(p, t) \quad O(p= \pm \infty, x= \pm \infty, t)=0
$$

\section{Reduce equation}

Define friction function by using bath potential:

$$
\gamma(t)=\sum_{j=1}^{N}\left(\frac{c_{j}}{\omega_{j}}\right)^{2} \cos \left(\omega_{j}\right)
$$

Friction $\gamma$ is zero if constant $c j=0$, that if the bath potential is zero or no bath potential, there is not friction for system. In the case frequent of bath is very large, the value of friction will be small. Under condition bath potential is classical, and the friction is Ohmic: $\gamma(t)=2 \gamma \delta(t)$, where $\gamma$ is the damping coefficient. Above Wigner-Liouville equation reduces $\mathrm{t}$

$$
\left.\frac{\partial O}{\partial t}=\left[p \frac{\partial}{\partial x}-\left(\not p+v^{\prime}\right) \frac{\partial}{\partial p}+\frac{\gamma}{\beta} \frac{\partial^{2}}{\partial p^{2}}\right)\right] O+W(p, x, t)
$$

The function $\mathrm{W}(\mathrm{p}, \mathrm{x}, \mathrm{t})$ depend on potential $\mathrm{v}(\mathrm{x})$. We consider two examples.

Example 1, harmonic potential $v(x)=x^{2} / 2$

We may calculate “quantum function"” $\mathrm{W}=0$.

Example 2 double well potential $v\left(x_{0}\right)=x_{0}{ }^{4} / 4-x_{0}{ }^{2}$

For the quartic potential, we have result:

$$
W(p, x, t)=x \frac{\partial^{3} O}{\partial p^{3}}
$$

\section{Difference formula}

Making space step is h, time step is $\tau$, $x i=i h, p j=j h, t k=k \tau, O i j(k)=O(x i, p j, t k), W i j(k)=W(x i, p j, t k)$. The control equation can be written as explicit numeric formula as below:

$$
\begin{aligned}
& O_{i j}^{(k+1)}=O_{i j}^{(k)}+\tau O_{i j}^{(k), 0} \\
& O_{i j}^{(k), 0}=\left\{p_{j} \frac{\partial}{\partial x}-\left[\gamma p_{j}+v^{\prime}\left(x_{i}\right)\right] \frac{\partial}{\partial p}+\frac{\gamma}{\beta} \frac{\partial^{2}}{\partial p^{2}}+x_{i} \frac{\partial^{3}}{\partial p^{3}}\right\} O_{i j}^{(k)}
\end{aligned}
$$

And

$$
O_{i j}^{(k+1)}=O_{i j}^{(k)}+\lambda O_{i j}^{(k), 0}+(1-\lambda) O_{i j}^{(k+1), 1}
$$

where the value of the parameter $\lambda$ is between zero and unity. Its value is determined by searching for that value at which we can take the largest integration step size while keeping the numerical accuracy sufficiently small.

\section{5 parallel calculation}

Take numeric domain $-\mathrm{L}<\mathrm{p}, \mathrm{x}<\mathrm{L}$ For the harmonic oscillator potential, we chose $\mathrm{Np}=1$, and for the quartic potential we chose $\mathrm{Np}=2$. The boundary $\mathrm{L}$ was determined by checking convergence of the results with respect to the magnitude of the boundary. Practically this meant using $\mathrm{L}=6$ for all computations.. 


\section{6 numeric results}

The numeric domain is decomposed into 20 sub-domains as $-6+0.6 n-0.6<x<-6+0.6 n,-L<p<L$, $\mathrm{n}=1,2, \ldots, 20$. Using a grid size of $\mathrm{h}=0.05$, his time step was $\mathrm{dt}=10-6$. We found that $\lambda=0.98$ for low friction and $\lambda=0.96$ for high friction were the optimal parameter values. Date of function is exchanged between sub-domains. In Fig. 1 we compare our results ( solid line ) with those of Stockburger and Mak for the harmonic oscillator for the temperatures $\mathrm{T}=0.2,2$ ( in units such that the Boltzmann constant is unity ) and the damping constant is $\gamma=0.2$. The initial value of the coordinate was chosen such that $<\mathrm{x} 2>=\mathrm{T}$. As may be seen, there is excellent agreement for both the high and the low temperature cases. This good agreement is really not very surprising. The Wigner-Fokker-Planck equation is expected to be good at the high temperature. Even at the low temperature case, the agreement comes as a result of the rather weak damping, so that the low temperature case is dominated by the system dynamics, which are treated exactly within the Wigner-Fokker-Planck equation. For this harmonic case, the Wigner-Fokker-Planck and the mixed quantum classical approximations, in which the classical dynamics are obtained from the Langevin equation, are identical.

\section{Summary}

he paper discusses a parallel explicit and implicit difference method to solve time dependent initial boundary condition quantum dynamics Wigner - Fokker - Planck (WFP) equation.The main result presented in this paper is that the WFP equation gives rather good agreement with numerically exact path integral results, obtained with factorized initial conditions. The WFP equation manages to treat well both coherence as well as tunneling phenomena. Numerical solution of the Wigner - Fokker Planck equation is order of magnitude faster that the path integral solution. All this implies that it is worthwhile to extend the present study of the WFP equation to other systems.

\section{References}

[1] C. H. Mak and R. Egger, Adv. Chem. Phys. 93, 39 1996.

[2] J. T. Stockburger and C. H. Mak, Chem. Phys. 268, $249 \sim 2001$.

[3] A. O. Caldeira and A. J. Leggett, Physica A 121, $587 \sim 1983$.

[4] G. Ashkenazi, U. Banin, A. Bartana, S. Ruhman, and R. Kosloff, Adv.

[5] Y. Tanimura and R. Kubo, J. Phys. Soc. Jpn. 58, $101 \sim 1989$ Chem. Phys. 100, $229 \sim 1997$.

[6] Pechukas, J. Ankerhold, and H. Grabert, Ann. Phys. Leipzig! 9, $794 \sim 2000$

[7] Yu Qing, Wu Xiaoyuan,Yuan Meng,Xiong Qian, Jin Shengping,The empirical analysis on the influential factors of urbanization in Hubei province based on the panel data[M], Proceeding of

DCABES 2015， 17-24Aug.2015， Guiyang, China., pp79-82.

[8] J. T. Stockburger and C. H. Mak, J. Chem. Phys. 110, 4983 1999. 


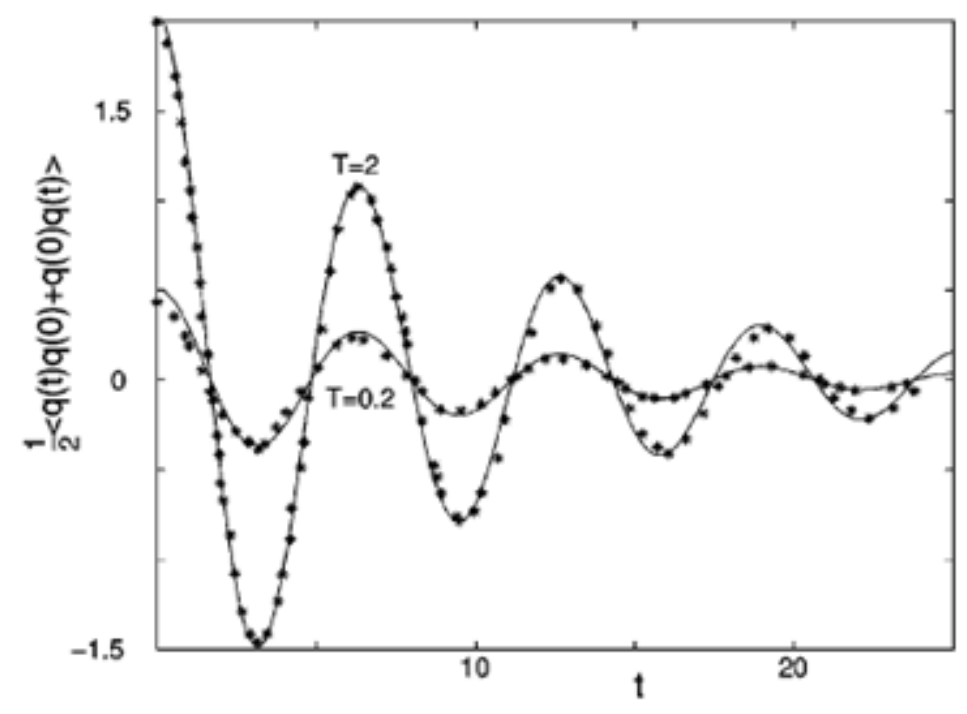

Figure 1. The position autocorrelation function for a dissipative harmonic oscillator. The stars are the numerically exact path integral results (Ref. 8), the solid lines are the results obtained from the Wigner-Fokker-Planck equation. The high temperature $(T=2)$ and low temperature $(T=0.2)$ results are those with large and small initial amplitude, respectively. The damping constant $\gamma=0.2$.

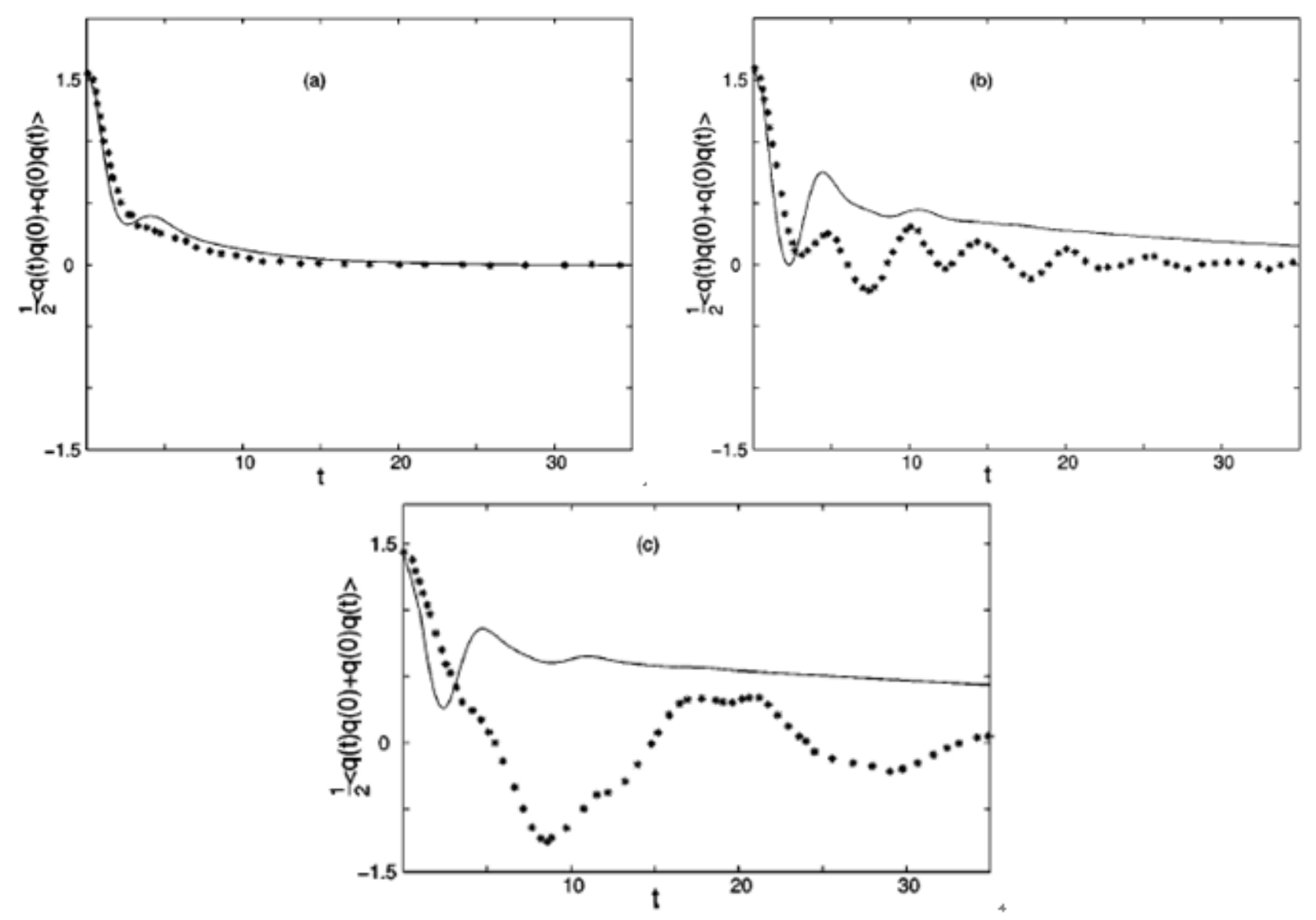

Figure 2. The position autocorrelation function for a symmetric quartic double well potential. The stars are the numerically exact path integral results $\sim$ Ref. 4 !, the solid lines are the results obtained from the mixed quantum classical theory. Panels $\sim a !-\sim c$ ! are for $(T=2, \gamma=0.5),(T=1, \gamma=0.05)$, and $(\mathrm{T}=0.5, \gamma=0.05)$, respectively. 